\title{
TRANSITION PROBABILITIES FOR FORBIDDEN LINES
}

\author{
R. H. Garstang \\ (Joint Institute for Laboratory Astrophysics* and University of Colorado, U.S.A.)
}

\begin{abstract}
A compilation is given of transition probabilities of forbidden lines which occur in the spectra of gaseous nebulae.

The spectra of gaseous nebulae contain both permitted and forbidden lines of many elements in various stages of ionization. These are of great importance because of the information they can provide on the physical conditions and chemical composition of the nebulae. The forbidden lines belong to both non-metals and metals, and indeed for some elements the only observable spectral lines are forbidden lines. Transition probabilities are now known for essentially every forbidden line which has been observed with reasonable certainty in gaseous nebulae, including the Orion Nebula and the very rich-spectrum planetary nebula NGC 7027.
\end{abstract}

Table 1

Transition probabilities ${ }^{* *}$ for the $2 \mathbf{p}^{2}$ configuration

\begin{tabular}{|c|c|c|c|c|c|}
\hline Transition & $\mathrm{CI}_{\mathrm{I}}$ & $\mathrm{N}_{I I}$ & OIII & Fiv & $\mathrm{NeV}$ \\
\hline${ }^{1} \mathrm{D}_{2}-{ }^{1} \mathrm{~S}_{0}$ & $\begin{array}{c}0.50 \\
8727 \cdot 4\end{array}$ & $\begin{array}{c}1.08 \\
5754 \cdot 6\end{array}$ & $\begin{array}{c}1 \cdot 60 \\
4363 \cdot 2\end{array}$ & $\begin{array}{c}2 \cdot 10 \\
3532 \cdot 2\end{array}$ & $\begin{array}{l}2 \cdot 60 \\
2972\end{array}$ \\
\hline${ }^{3} \mathbf{P}_{2}-{ }^{-1} \mathbf{S}_{0}$ & $\begin{array}{c}1.9 \times 10^{-5} \\
4627.3\end{array}$ & $\begin{array}{c}1.6 \times 10^{-4} \\
3070.8\end{array}$ & $\begin{array}{c}7 \cdot 1 \times 10^{-4} \\
2331.6\end{array}$ & $\begin{array}{c}2.3 \times 10^{-3} \\
1889.3\end{array}$ & $\begin{array}{c}6.8 \times 10^{-3} \\
1592.7\end{array}$ \\
\hline${ }^{3} \mathbf{P}_{1}-{ }^{1} \mathbf{S}_{0}$ & $\begin{array}{c}2.6 \times 10^{-3} \\
4621 \cdot 5\end{array}$ & $\begin{array}{c}0.034 \\
3063 \cdot 0\end{array}$ & $\begin{array}{c}0 \cdot 23 \\
2321 \cdot 1\end{array}$ & $\begin{array}{c}1 \cdot 1 \\
1875 \cdot 5\end{array}$ & $\begin{array}{c}4 \cdot 2 \\
1575 \cdot 2\end{array}$ \\
\hline${ }^{3} \mathbf{P}_{2}-{ }^{1} D_{2}$ & $\begin{array}{c}2.3 \times 10^{-4} \\
9849.5\end{array}$ & $\begin{array}{c}3.0 \times 10^{-3} \\
6583.4\end{array}$ & $\begin{array}{c}0.021 \\
5006 \cdot 8\end{array}$ & $\begin{array}{c}0.098 \\
4060 \cdot 2\end{array}$ & $\begin{array}{c}0.38 \\
3425 \cdot 9\end{array}$ \\
\hline${ }^{3} \mathbf{P}_{1}-{ }^{1} \mathbf{D}_{2}$ & $\begin{array}{c}7.8 \times 10^{-5} \\
9823.4\end{array}$ & $\begin{array}{c}1.03 \times 10^{-3} \\
6548.1\end{array}$ & $\begin{array}{l}0.0071 \\
4958.9\end{array}$ & $\begin{array}{c}0.034 \\
3997 \cdot 4\end{array}$ & $\begin{array}{c}0.138 \\
3345 \cdot 8\end{array}$ \\
\hline${ }^{3} \mathbf{P}_{0}-{ }^{1} \mathrm{D}_{2}$ & $\begin{array}{c}5.5 \times 10^{-8} \\
9808.9\end{array}$ & $\begin{array}{c}4.2 \times 10^{-7} \\
6527.4\end{array}$ & $\begin{array}{c}1.9 \times 10^{-6} \\
4931.0\end{array}$ & $\begin{array}{c}6.4 \times 10^{-6} \\
3960.7\end{array}$ & $\begin{array}{c}1.9 \times 10^{-5} \\
3300.0\end{array}$ \\
\hline${ }^{3} \mathbf{P}_{1}-{ }^{3} \mathbf{P}_{2}$ & $2.7 \times 10^{-7}$ & $7.5 \times 10^{-6}$ & $9.8 \times 10^{-5}$ & $7.9 \times 10^{-4}$ & $4.6 \times 10^{-3}$ \\
\hline${ }^{3} P_{0}-{ }^{3} P_{2}$ & $2.0 \times 10^{-14}$ & $1.3 \times 10^{-12}$ & $3.5 \times 10^{-11}$ & $5.0 \times 10^{-10}$ & $5 \cdot 2 \times 10^{-9}$ \\
\hline${ }^{3} P_{0}-{ }^{3} P_{1}$ & $7.9 \times 10^{-8}$ & $2.1 \times 10^{-6}$ & $2.6 \times 10^{-5}$ & $2.1 \times 10^{-4}$ & $1.3 \times 10^{-3}$ \\
\hline
\end{tabular}

** Compiled from Wiese et al. (1966), which is based on work by Garstang; Naqvi ; and Yamanouchi and Horie.

* Of the National Bureau of Standards and the University of Colorado.

Osterbrock and O'Dell (eds.), Planetary Nebulae, 143-152. O I.A.U. 
Table 2

\section{Transition probabilities* for the $2 \mathrm{p}^{3}$ configuration}

\begin{tabular}{|c|c|c|c|c|c|}
\hline Transition & $\mathrm{N}_{\mathrm{I}}$ & $\mathrm{O}_{\text {II }}$ & F III & NeIV & $\mathrm{Mg}$ vI \\
\hline${ }^{2} \mathbf{P}_{1}-2 \mathbf{P}_{1_{\frac{1}{2}}}$ & (very small) & $6.0 \times 10^{-11}$ & (very small) & $2.3 \times 10^{-9}$ & $1.6 \times 10^{-5}$ \\
\hline${ }^{2} \mathbf{D}_{2 \frac{1}{3}}-{ }^{2} \mathbf{P}_{1 \frac{1}{2}}$ & $\begin{array}{c}0.054 \\
10395 \cdot 4\end{array}$ & $\begin{array}{c}0.115 \\
7319 \cdot 4\end{array}$ & $\begin{array}{c}0.18 \\
5721 \cdot 2\end{array}$ & $\begin{array}{c}0.40 \\
4714 \cdot 3\end{array}$ & $\begin{array}{c}2 \cdot 4 \\
3485 \cdot 5\end{array}$ \\
\hline${ }^{2} \mathbf{D}_{1 !}-{ }^{2} \mathbf{P}_{1 !}$ & $\begin{array}{c}0.025 \\
10404 \cdot 1\end{array}$ & $\begin{array}{c}0.061 \\
7330.7\end{array}$ & $\begin{array}{c}0 \cdot 114 \\
5733 \cdot 0\end{array}$ & $\begin{array}{c}0.44 \\
4724 \cdot 2\end{array}$ & $\begin{array}{c}3 \cdot 8 \\
3488 \cdot 1\end{array}$ \\
\hline${ }^{2} \mathrm{D}_{2}-{ }^{2} \mathrm{P}_{1}$ & $\begin{array}{c}0.031 \\
10395.4\end{array}$ & $\begin{array}{c}0.061 \\
7318 \cdot 6\end{array}$ & $\begin{array}{c}0.088 \\
5721 \cdot 2\end{array}$ & $\begin{array}{c}0 \cdot 11 \\
4715 \cdot 6\end{array}$ & $\begin{array}{c}0.15 \\
3500 \cdot 4\end{array}$ \\
\hline${ }^{2} \mathbf{D}_{1 \frac{1}{2}}-{ }^{2} \mathbf{P}_{\frac{1}{2}}$ & $\begin{array}{c}0.047 \\
10404 \cdot 1\end{array}$ & $\begin{array}{c}0.100 \\
7329.9\end{array}$ & $\begin{array}{c}0 \cdot 16 \\
5733 \cdot 0\end{array}$ & $\begin{array}{c}0.39 \\
4725 \cdot 6\end{array}$ & $\begin{array}{c}2 \cdot 5 \\
3503 \cdot 0\end{array}$ \\
\hline${ }^{4} \mathbf{S}_{1_{\frac{1}{2}}-2} \mathbf{P}_{1 \frac{1}{1}}$ & $\begin{array}{c}6.2 \times 10^{-3} \\
3466.4\end{array}$ & $\begin{array}{c}0.060 \\
2470.4\end{array}$ & $\begin{array}{c}0.26 \\
1939 \cdot 6\end{array}$ & $\begin{array}{c}1 \cdot 33 \\
1608 \cdot 8\end{array}$ & 13 \\
\hline${ }^{4} S_{1_{1}}-{ }^{2} P_{\frac{1}{2}}$ & $\begin{array}{c}2.5 \times 10^{-3} \\
3466.4\end{array}$ & $\begin{array}{l}0.0238 \\
2470 \cdot 3\end{array}$ & $\begin{array}{c}0 \cdot 10 \\
1939 \cdot 6\end{array}$ & $\begin{array}{c}0.53 \\
1609.0\end{array}$ & $5 \cdot 3$ \\
\hline${ }^{2} \mathrm{D}_{2 !}-{ }^{2} \mathrm{D}_{1 \xi}$ & $1.3 \times 10^{-8}$ & $1.3 \times 10^{-7}$ & $7.6 \times 10^{-7}$ & $1.4 \times 10^{-6}$ & $1.5 \times 10^{-7}$ \\
\hline${ }^{4} \mathrm{~S}_{1 !}-{ }^{2} \mathrm{D}_{2 !}$ & $\begin{array}{c}6.9 \times 10^{-6} \\
5200 \cdot 4\end{array}$ & $\begin{array}{c}4.8 \times 10^{-5} \\
3728.8\end{array}$ & $\begin{array}{c}1 \cdot 3 \times 10^{-4} \\
2933 \cdot 1\end{array}$ & $\begin{array}{c}5.9 \times 10^{-4} \\
2441 \cdot 3\end{array}$ & 0.0054 \\
\hline${ }^{4} S_{1 !}-{ }^{2} D_{1 !}$ & $\begin{array}{c}1.6 \times 10^{-5} \\
5197.9\end{array}$ & $\begin{array}{c}1.70 \times 10^{-4} \\
3726.0\end{array}$ & $\begin{array}{c}1.3 \times 10^{-3} \\
2930.0\end{array}$ & $\begin{array}{c}5.6 \times 10^{-3} \\
2438.6\end{array}$ & 0.12 \\
\hline
\end{tabular}

* Compiled for Ni, OII, FiII and NeIv from Wiese et al. (1966), which is based on work by Ufford and Gilmour; Naqvi; Garstang; and Seaton and Osterbrock. Mgvi recalculated by Garstang for inclusion here.

Table 3

\section{Transition probabilities* for the $2 \mathbf{p}^{4}$ configuration}

\begin{tabular}{|c|c|c|c|c|}
\hline Transition & $\mathrm{OI}_{\mathrm{I}}$ & FII & $\mathrm{Ne}$ III & NaIV \\
\hline${ }^{1} \mathbf{D}_{2}-{ }^{-1} \mathbf{S}_{0}$ & $\begin{array}{c}1 \cdot 34 \\
5577 \cdot 4\end{array}$ & $\begin{array}{c}2 \cdot 1 \\
4157 \cdot 5\end{array}$ & $\begin{array}{c}2 \cdot 8 \\
3342 \cdot 5\end{array}$ & $\begin{array}{c}3 \cdot 5 \\
2803 \cdot 3\end{array}$ \\
\hline${ }^{3} \mathbf{P}_{2}-{ }^{-1} S_{0}$ & $\begin{array}{c}3.7 \times 10^{-4} \\
2958.4\end{array}$ & $\begin{array}{c}1.6 \times 10^{-3} \\
2225.5\end{array}$ & $\begin{array}{c}5 \cdot 1 \times 10^{-3} \\
1793.8\end{array}$ & $\begin{array}{c}0.012 \\
1503.7\end{array}$ \\
\hline${ }^{3} \mathbf{P}_{1}-{ }^{1} \mathbf{S}_{0}$ & $\begin{array}{c}0.067 \\
2972 \cdot 3\end{array}$ & $\begin{array}{c}0.49 \\
2246 \cdot 6\end{array}$ & $\begin{array}{c}2 \cdot 2 \\
1814 \cdot 8\end{array}$ & $\begin{array}{c}7 \cdot 6 \\
1529 \cdot 1\end{array}$ \\
\hline${ }^{3} P_{2^{-}}{ }^{1} D_{2}$ & $\begin{array}{c}5.1 \times 10^{-3} \\
6300 \cdot 3\end{array}$ & $\begin{array}{c}0.038 \\
4789.5\end{array}$ & $\begin{array}{c}0.17 \\
3868 \cdot 8\end{array}$ & $\begin{array}{c}0.66 \\
3241 \cdot 7\end{array}$ \\
\hline${ }^{3} P_{1}-{ }^{1} D_{2}$ & $\begin{array}{c}1.64 \times 10^{-3} \\
6363.8\end{array}$ & $\begin{array}{c}0.012 \\
4869 \cdot 3\end{array}$ & $\begin{array}{c}0.052 \\
3967.5\end{array}$ & $\begin{array}{c}0.20 \\
3362 \cdot 2\end{array}$ \\
\hline${ }^{3} P_{0}-{ }^{-1} D_{2}$ & $\begin{array}{c}1.1 \times 10^{-6} \\
6391.6\end{array}$ & $\begin{array}{c}4.1 \times 10^{-6} \\
4904.8\end{array}$ & $\begin{array}{c}1.2 \times 10^{-5} \\
4012.7\end{array}$ & $\begin{array}{c}3.0 \times 10^{-5} \\
3416 \cdot 2\end{array}$ \\
\hline${ }^{3} \mathbf{P}_{1}-{ }^{3} \mathbf{P}_{0}$ & $1.7 \times 10^{-5}$ & $1.8 \times 10^{-4}$ & $1.2 \times 10^{-3}$ & $5.5 \times 10^{-3}$ \\
\hline${ }^{3} \mathbf{P}_{2^{-}}{ }^{-3} \mathbf{P}_{0}$ & $1.0 \times 10^{-10}$ & $1.8 \times 10^{-9}$ & $2.0 \times 10^{-8}$ & $1.5 \times 10^{-7}$ \\
\hline${ }^{3} \mathbf{P}_{2}-{ }^{3} \mathbf{P}_{1}$ & $9.0 \times 10^{-5}$ & $9.0 \times 10^{-4}$ & $6.0 \times 10^{-3}$ & 0.030 \\
\hline
\end{tabular}

* Compiled for OI, FII and NeIII from Wiese et al. (1966), based on work by Garstang; Naqvi; Yamanouchi and Horie and for $\left(\mathrm{O}_{\mathrm{I}}\right)$ Omholt; Stoffregen and Derblom. NaIv from Malville and Berger (1965). 


\section{Table 4}

\section{Transition probabilities* for the $3 p^{2}$ configuration}

\begin{tabular}{|c|c|c|c|c|c|}
\hline Transition & SIII & Cliv & Arv & K vI & CaviI \\
\hline${ }^{1} \mathrm{D}_{2}{ }^{-1} \mathrm{~S}_{0}$ & $\begin{array}{c}2 \cdot 54 \\
6312 \cdot 1\end{array}$ & $\begin{array}{c}3 \cdot 15 \\
5323 \cdot 3\end{array}$ & $\begin{array}{c}3.78 \\
4625 \cdot 5\end{array}$ & $\begin{array}{c}4 \cdot 1 \\
4097:\end{array}$ & $\begin{array}{c}4 \cdot 3 \\
3688:\end{array}$ \\
\hline${ }^{3} \mathbf{P}_{2}-{ }^{-1} \mathbf{S}_{0}$ & $\begin{array}{c}0.016 \\
3796 \cdot 7\end{array}$ & $\begin{array}{c}0.038 \\
3203 \cdot 2\end{array}$ & $\begin{array}{c}0.081 \\
2784 \cdot 4\end{array}$ & $\begin{array}{c}0 \cdot 14 \\
2471 \cdot 7\end{array}$ & $\begin{array}{c}0.25 \\
2226:\end{array}$ \\
\hline${ }^{3} \mathbf{P}_{1}-{ }^{-1} \mathbf{S}_{0}$ & $\begin{array}{c}0.85 \\
3721.7\end{array}$ & $\begin{array}{c}2 \cdot 6 \\
3118 \cdot 3\end{array}$ & $\begin{array}{c}6 \cdot 8 \\
2691 \cdot 4\end{array}$ & $\begin{array}{c}16 \cdot \\
2366 \cdot 8\end{array}$ & $\begin{array}{l}34 . \\
2112:\end{array}$ \\
\hline${ }^{3} P_{2}-{ }^{1} D_{2}$ & $\begin{array}{c}0.064 \\
9532 \cdot 1\end{array}$ & $\begin{array}{c}0.20 \\
8045 \cdot 6\end{array}$ & $\begin{array}{c}0.51 \\
7005 \cdot 7\end{array}$ & $\begin{array}{c}1 \cdot 1 \\
6228 \cdot 4\end{array}$ & $\begin{array}{c}2 \cdot 5 \\
5614 \cdot 7\end{array}$ \\
\hline${ }^{3} P_{1}-{ }^{1} D_{2}$ & $\begin{array}{c}0.025 \\
9069 \cdot 4\end{array}$ & $\begin{array}{c}0.080 \\
7530.5\end{array}$ & $\begin{array}{c}0.22 \\
6435 \cdot 1\end{array}$ & $\begin{array}{c}0.53 \\
5603 \cdot 2\end{array}$ & $\begin{array}{c}1 \cdot 2 \\
4939:\end{array}$ \\
\hline${ }^{3} \mathrm{P}_{0^{-}}{ }^{1} \mathrm{D}_{2}$ & $\begin{array}{c}9.1 \times 10^{-6} \\
8831.5\end{array}$ & $\begin{array}{c}2.2 \times 10^{-5} \\
7262.3\end{array}$ & $\begin{array}{c}4.9 \times 10^{-5} \\
6131.0\end{array}$ & $\begin{array}{c}1.1 \times 10^{-4} \\
5269.2\end{array}$ & $\begin{array}{c}2 \cdot 1 \times 10^{-4} \\
4571:\end{array}$ \\
\hline${ }^{3} \mathbf{P}_{1}-{ }^{-3} \mathbf{P}_{2}$ & $2.4 \times 10^{-3}$ & $8.2 \times 10^{-3}$ & 0.027 & 0.076 & 0.20 \\
\hline${ }^{3} \mathbf{P}_{0}-{ }^{-3} P_{2}$ & $4.7 \times 10^{-8}$ & $2.8 \times 10^{-7}$ & $1.3 \times 10^{-6}$ & $5.4 \times 10^{-6}$ & $1.9 \times 10^{-5}$ \\
\hline${ }^{2} \mathbf{P}_{0}-{ }^{3} P_{1}$ & $4.7 \times 10^{-4}$ & $2.1 \times 10^{-3}$ & $8.0 \times 10^{-3}$ & 0.026 & 0.076 \\
\hline
\end{tabular}

* SiII, Cliv and Arv from Czyzak and Krueger (1963), K vi and Cavir from Malville and Berger (1965).

\section{Table 5}

\section{Transition probabilities* for the $3 p^{3}$ configuration}

\begin{tabular}{|c|c|c|c|c|}
\hline Transition & $S_{\text {II }}$ & CliII & ArIv & $\mathrm{KV}$ \\
\hline${ }^{2} P_{\frac{1}{1}}-{ }^{2} P_{1 !}$ & $1.0 \times 10^{-6}$ & $7.6 \times 10^{-6}$ & $5.2 \times 10^{-5}$ & $2.8 \times 10^{-4}$ \\
\hline${ }^{2} \mathbf{D}_{2 \xi}-{ }^{-2} P_{1 \xi}$ & $\begin{array}{c}0.21 \\
10320 \cdot 6\end{array}$ & $\begin{array}{c}0.36 \\
8481 \cdot 6\end{array}$ & $\begin{array}{c}0.67 \\
7237 \cdot 3\end{array}$ & $\begin{array}{c}1.5 \\
6317:\end{array}$ \\
\hline${ }^{2} D_{1 \frac{1}{2}}-{ }^{2} P_{1 \frac{1}{2}}$ & $\begin{array}{c}0.17 \\
10287 \cdot 1\end{array}$ & $\begin{array}{c}0.39 \\
8433.7\end{array}$ & $\begin{array}{c}0.91 \\
7170 \cdot 6\end{array}$ & $\begin{array}{c}2 \cdot 3 \\
6223:\end{array}$ \\
\hline${ }^{2} D_{2 !}-2 P_{t}$ & $\begin{array}{c}0.087 \\
10372 \cdot 6\end{array}$ & $\begin{array}{c}0.108 \\
8550 \cdot 5\end{array}$ & $\begin{array}{c}0.122 \\
7332 \cdot 0\end{array}$ & $\begin{array}{l}0.19 \\
6447:\end{array}$ \\
\hline${ }^{2} D_{1,}-2 P_{t}$ & $\begin{array}{c}0 \cdot 20 \\
10338 \cdot 8\end{array}$ & $\begin{array}{c}0.35 \\
8501 \cdot 8\end{array}$ & $\begin{array}{c}0.68 \\
7262 \cdot 8\end{array}$ & $\begin{array}{c}1.5 \\
6349:\end{array}$ \\
\hline${ }^{4} S_{1 \xi}-{ }^{-2} P_{1 \xi}$ & $\begin{array}{c}0.34 \\
4068 \cdot 6\end{array}$ & $\begin{array}{c}0.96 \\
3342 \cdot 9\end{array}$ & $\begin{array}{c}2.55 \\
2854 \cdot 8\end{array}$ & $\begin{array}{c}6 \cdot 5 \\
2494 \cdot 5\end{array}$ \\
\hline${ }^{4} S_{1 !}-{ }^{2} P_{t}$ & $\begin{array}{c}0 \cdot 134 \\
4076 \cdot 4\end{array}$ & $\begin{array}{c}0.37 \\
3353 \cdot 3\end{array}$ & $\begin{array}{c}0.97 \\
2869 \cdot 1\end{array}$ & $\begin{array}{c}2 \cdot 4 \\
2514 \cdot 5\end{array}$ \\
\hline${ }^{2} D_{2 t}-{ }^{2} D_{1 \xi}$ & $3.3 \times 10^{-7}$ & $3.2 \times 10^{-6}$ & $2 \cdot 3 \times 10^{-5}$ & $1.4 \times 10^{-4}$ \\
\hline${ }^{4} S_{1 !}-{ }^{2} D_{2 \xi}$ & $\begin{array}{c}4.7 \times 10^{-5} \\
6716.4\end{array}$ & $\begin{array}{c}1.01 \times 10^{-3} \\
5517.2\end{array}$ & $\begin{array}{c}2.2 \times 10^{-3} \\
4711.3\end{array}$ & $\begin{array}{c}6.9 \times 10^{-3} \\
4122.6\end{array}$ \\
\hline${ }^{4} \mathbf{S}_{1,}-{ }^{2} \mathbf{D}_{1 \xi}$ & $\begin{array}{c}3.0 \times 10^{-4} \\
6730.8\end{array}$ & $\begin{array}{c}7.0 \times 10^{-3} \\
5537.7\end{array}$ & $\begin{array}{c}0.028 \\
4740 \cdot 2\end{array}$ & $\begin{array}{c}0 \cdot 11 \\
4163 \cdot 3\end{array}$ \\
\hline
\end{tabular}

* From Czyzak and Krueger (1963) for SII, CliII and ArIv. Kv calculated by Garstang for inclusion here. 
Table 6

\section{Transition probabilities ${ }^{*}$ for the $3 \mathbf{p}^{4}$ configuration}

\begin{tabular}{|c|c|c|c|c|c|}
\hline Transition & $S_{I}$ & $\mathrm{Cl}_{\text {II }}$ & ArIII & KIV & $\mathrm{Cav}$ \\
\hline${ }^{1} \mathrm{D}_{2}{ }^{-1} \mathrm{~S}_{0}$ & $\begin{array}{c}1.78 \\
7724 \cdot 7\end{array}$ & $\begin{array}{c}2.3 \\
6152.9\end{array}$ & $\begin{array}{c}3 \cdot 1 \\
5191.8\end{array}$ & $\begin{array}{c}3.9 \\
4510.9\end{array}$ & $\begin{array}{c}4 \cdot 6 \\
3996 \cdot 3\end{array}$ \\
\hline${ }^{3} \mathbf{P}_{2}-{ }^{-1} \mathbf{S}_{0}$ & $\begin{array}{c}7.3 \times 10^{-3} \\
4506.9\end{array}$ & $\begin{array}{c}0.018 \\
3583 \cdot 0\end{array}$ & $\begin{array}{c}0.043 \\
3005 \cdot 1\end{array}$ & $\begin{array}{c}0.086 \\
2593.5\end{array}$ & $\begin{array}{c}0 \cdot 16 \\
2280 \cdot 0\end{array}$ \\
\hline${ }^{3} \mathbf{P}_{1}-{ }^{-1} \mathbf{S}_{0}$ & $\begin{array}{c}0.35 \\
4589.0\end{array}$ & $\begin{array}{c}1 \cdot 3 \\
3675 \cdot 0\end{array}$ & $\begin{array}{c}4.0 \\
3109.0\end{array}$ & $\begin{array}{c}10 \cdot 4 \\
2711 \cdot 2\end{array}$ & $\begin{array}{c}24 \cdot \\
2412 \cdot 4\end{array}$ \\
\hline${ }^{3} \mathbf{P}_{2}{ }^{-1} \mathbf{D}_{2}$ & $\begin{array}{c}0.028 \\
10819.8\end{array}$ & $\begin{array}{c}0.10 \\
8579.5\end{array}$ & $\begin{array}{c}0.32 \\
7135 \cdot 8\end{array}$ & $\begin{array}{c}0.83 \\
6101 \cdot 8\end{array}$ & $\begin{array}{c}1.9 \\
5309.2\end{array}$ \\
\hline${ }^{3} \mathbf{P}_{1}-{ }^{1} D_{2}$ & $\begin{array}{c}8.0 \times 10^{-3} \\
11305.8\end{array}$ & $\begin{array}{c}0.029 \\
9125 \cdot 8\end{array}$ & $\begin{array}{c}0.083 \\
7751.0\end{array}$ & $\begin{array}{c}0 \cdot 20 \\
6795 \cdot 8\end{array}$ & $\begin{array}{c}0.43 \\
6086 \cdot 9\end{array}$ \\
\hline${ }^{3} \mathrm{P}_{0}-{ }^{-1} \mathrm{D}_{2}$ & $\begin{array}{c}5 \cdot 0 \times 10^{-6} \\
11540 \cdot 1\end{array}$ & $\begin{array}{c}1.2 \times 10^{-5} \\
9381.8\end{array}$ & $\begin{array}{c}2.9 \times 10^{-5} \\
8036.4\end{array}$ & $\begin{array}{c}6.0 \times 10^{-5} \\
7110.4\end{array}$ & $\begin{array}{c}1.1 \times 10^{-4} \\
6428.2\end{array}$ \\
\hline${ }^{3} \mathbf{P}_{1}-{ }^{3} \mathbf{P}_{0}$ & $3.0 \times 10^{-4}$ & $1.4 \times 10^{-3}$ & $5 \cdot 1 \times 10^{-3}$ & 0.015 & 0.035 \\
\hline${ }^{3} \mathbf{P}_{2^{-}}{ }^{3} \mathbf{P}_{0}$ & $7 \cdot 1 \times 10^{-8}$ & $4.8 \times 10^{-7}$ & $2.7 \times 10^{-6}$ & $1.2 \times 10^{-5}$ & $4.5 \times 10^{-5}$ \\
\hline${ }^{3} \mathbf{P}_{2}-{ }^{3} \mathbf{P}_{1}$ & $1.4 \times 10^{-3}$ & $7.5 \times 10^{-3}$ & 0.031 & $0 \cdot 10$ & 0.31 \\
\hline
\end{tabular}

* Si, Clir and Ar III from Czyzak and Krueger (1963), K IV and Ca v from Malville and Berger (1965).

Table 7

Transition probabilities for selected* lines of Mnvi and FevII

\begin{tabular}{|c|c|c|c|c|c|}
\hline \multirow{2}{*}{ Transition } & \multirow{2}{*}{$\mathbf{J}-\mathbf{J}^{\prime}$} & \multicolumn{2}{|c|}{ MnvI } & \multicolumn{2}{|c|}{ Fevil } \\
\hline & & $\lambda$ & $A$ & $\lambda$ & $A$ \\
\hline \multirow[t]{3}{*}{$a^{3} F-a^{1} D$} & $2-2$ & $6518 \cdot 3$ & $0 \cdot 14$ & $5721 \cdot 1$ & $0 \cdot 30$ \\
\hline & $3-2$ & 6852: & $0 \cdot 23$ & $6086 \cdot 9$ & 0.49 \\
\hline & 4-2 & 7315: & $9.0 \times 10^{-4}$ & $6598 \cdot 8$ & $1.6 \times 10^{-3}$ \\
\hline \multirow[t]{6}{*}{$a^{3} F-a^{3} P$} & $2-0$ & 5622: & 0.087 & 4989: & $0 \cdot 11$ \\
\hline & $2-1$ & 5536: & 0.031 & 4893.4 & 0.043 \\
\hline & $3-1$ & $5776 \cdot 4$ & 0.050 & 5159.0 & 0.063 \\
\hline & $2-2$ & 5367: & $6.3 \times 10^{-3}$ & $4699 \cdot 8$ & 0.012 \\
\hline & $3-2$ & 5591: & 0.030 & $4944 \cdot 0$ & 0.065 \\
\hline & $4-2$ & 5894.0 & 0.050 & $5277 \cdot 7$ & 0.060 \\
\hline \multirow[t]{2}{*}{$a^{3} F-a^{1} G$} & $3-4$ & $4036 \cdot 8$ & 0.12 & $3587 \cdot 8$ & 0.26 \\
\hline & $4-4$ & $4193 \cdot 1$ & $0 \cdot 17$ & $3760 \cdot 3$ & 0.37 \\
\hline
\end{tabular}

* From Garstang (1964) for MnVI and Pasternack (1940) for Fe VII, with the electric quadrupole contributions in Fe vil reduced by the appropriate factor given by Garstang (1964). Results for many additional lines can be found in these references. 
Tables 1-11 give the transition probabilities of spontaneous emission in $\sec ^{-1}$. The transitions (with one exception specially noted) take place by magnetic dipole radiation, by electric quadrupole radiation, or by both. When both are possible one type usually predominates, but there are some cases where the two types of radiation have comparable probabilities. In our tables we have given the total transition probability; anyone interested in the type of radiation involved in a particular line and in the individual magnetic dipole or electric quadrupole-transition probabilities is referred to the original papers or compilations mentioned in the notes to the tables. The wavelengths are given (in Ångstroms unless microns are indicated) for lines in the observable spectral region and for some infrared and ultraviolet transitions. Many of the

\section{Table 8}

\section{Transition probabilities for selected* lines of Mnv and Fe vI}

\begin{tabular}{|c|c|c|c|c|c|}
\hline \multirow[b]{2}{*}{ Transition } & \multirow[b]{2}{*}{$\mathbf{J}-\mathbf{J}^{\prime}$} & \multicolumn{2}{|c|}{$\mathrm{Mnv}$} & \multicolumn{2}{|c|}{$\mathrm{Fe} \mathrm{vI}_{\mathrm{I}}$} \\
\hline & & $\lambda$ & $A$ & $\lambda$ & $A$ \\
\hline \multirow[t]{9}{*}{$a^{4} F-a^{4} P$} & $4 \frac{1}{2}-2 \frac{1}{2}$ & $6393 \cdot 6$ & 0.041 & $5677 \cdot 0$ & 0.048 \\
\hline & $3 \frac{1}{2}-2 \frac{1}{2}$ & $6166 \cdot 2$ & 0.016 & $5426 \cdot 6$ & 0.021 \\
\hline & $2 \frac{1}{2}-2 \frac{1}{2}$ & 5991: & $4 \cdot 1 \times 10^{-3}$ & 5233.9 & $5.9 \times 10^{-3}$ \\
\hline & $1 \frac{1}{2}-2 \frac{1}{2}$ & 5868: & $5.0 \times 10^{-4}$ & $5097 \cdot 5$ & $7.9 \times 10^{-4}$ \\
\hline & $3 \frac{1}{2}-1 \frac{1}{2}$ & $6346:$ & 0.031 & $5630 \cdot 8$ & 0.036 \\
\hline & $2 \frac{1}{2}-1 \frac{1}{2}$ & 6159: & 0.026 & 5423.9 & 0.032 \\
\hline & $1 \frac{1}{2}-1 \frac{1}{2}$ & 6030: & $9.3 \times 10^{-3}$ & 5277.5 & 0.014 \\
\hline & $2 \frac{1}{2}-\frac{1}{2}$ & $6218 \cdot 6$ & 0.026 & $5484 \cdot 8$ & 0.031 \\
\hline & $1 \frac{1}{2}-\frac{1}{2}$ & 6088: & 0.044 & $5335 \cdot 2$ & 0.055 \\
\hline \multirow[t]{7}{*}{$a^{4} F-a^{2} G$} & $4 \frac{1}{2}-4 \frac{1}{2}$ & $5891 \cdot 1$ & 0.24 & $5176 \cdot 4$ & 0.56 \\
\hline & $3 \frac{1}{2}-4 \frac{1}{2}$ & 5695: & 0.096 & $4967 \cdot 3$ & 0.22 \\
\hline & $2 \frac{1}{2}-4 \frac{1}{2}$ & 5544: & $1.7 \times 10^{-6}$ & $4805 \cdot 4$ & $3.1 \times 10^{6}$ \\
\hline & $4 \frac{1}{2}-3 \frac{1}{2}$ & 6069: & $5.9 \times 10^{-3}$ & 5370.5 & 0.012 \\
\hline & $3 \frac{1}{2}-3 \frac{1}{2}$ & $5862 \cdot 3$ & 0.096 & $5145 \cdot 8$ & $0 \cdot 22$ \\
\hline & $2 \frac{1}{2}-3 \frac{1}{2}$ & 5703: & 0.088 & $4972 \cdot 1$ & $0 \cdot 20$ \\
\hline & $1 \frac{1}{2}-3 \frac{1}{2}$ & 5592: & $5.6 \times 10^{-6}$ & $4849 \cdot 0$ & $1.1 \times 10^{-5}$ \\
\hline
\end{tabular}

* From Pasternack (1940) with the electric quadrupole contributions reduced by appropriate factors given by Garstang (1964). There are many other lines of these ions for which data can be found in these references.

wavelengths are quoted from Bowen (1955, 1960). Some wavelengths are uncertain by several tenths of an Ångstrom in cases where they have not been directly observed and reliance is upon predictions based on ultraviolet permitted-line spectroscopy.

A compilation of data for all atoms up to neon has been given by Wiese et al. (1966). Where the data we quote are the same as theirs, we reference only their book. References may be found in their book to the original papers by Garstang; Naqvi; Yamanouchi and Horie; Ufford and Gilmour; Seaton and Osterbrock; Omholt; and Stoffregen and Derblom upon which their compilation is based. For atoms heavier than 
Table 9

Transition probabilities for selected* lines of Fe III and Fev

\begin{tabular}{|c|c|c|c|c|c|}
\hline \multirow{2}{*}{ Transition } & \multirow{2}{*}{$\mathbf{J}-\mathbf{J}^{\prime}$} & \multicolumn{2}{|c|}{ FeIII } & \multicolumn{2}{|c|}{$\mathrm{FeV}$} \\
\hline & & $\lambda$ & $A$ & $\lambda$ & $A$ \\
\hline$a^{5} D-a^{5} D$ & $\begin{array}{l}0-1 \\
1-2 \\
2-3 \\
3-4\end{array}$ & & $\begin{array}{l}1.4 \times 10^{-4} \\
6.7 \times 10^{-4} \\
1.8 \times 10^{-3} \\
2.8 \times 10^{-3}\end{array}$ & & $\begin{array}{l}1.6 \times 10^{-4} \\
1.2 \times 10^{-3} \\
2.6 \times 10^{-3} \\
3.0 \times 10^{-3}\end{array}$ \\
\hline$a^{5} D-a^{3} P$ & $\begin{array}{l}1-0 \\
2-0 \\
0-1 \\
1-1 \\
2-1 \\
3-1 \\
0-2 \\
1-2 \\
2-2 \\
3-2 \\
4-2\end{array}$ & $\begin{array}{l}4930 \cdot 5 \\
4884 \cdot 5 \\
5084 \cdot 8 \\
5060 \cdot 5 \\
5011 \cdot 3 \\
4936 \cdot 4 \\
5439.9 \\
5412 \cdot 2 \\
5355 \cdot 9 \\
5270 \cdot 3 \\
5151.9\end{array}$ & $\begin{array}{l}0.67 \\
2.4 \times 10^{-4} \\
0.091 \\
1.5 \times 10^{-4} \\
0.53 \\
3.2 \times 10^{-5} \\
1.5 \times 10^{-5} \\
0.038 \\
1.1 \times 10^{-4} \\
0.40 \\
7.1 \times 10^{-6}\end{array}$ & $\begin{array}{l}4180 \cdot 9 \\
4229 \cdot 3 \\
4003 \cdot 0 \\
4026 \cdot 4 \\
4071 \cdot 3 \\
4136 \cdot 2 \\
3777 \cdot 2 \\
3798 \cdot 0 \\
3838 \cdot 0 \\
3895 \cdot 5 \\
3970 \cdot 0\end{array}$ & $\begin{array}{l}1.3 \\
2.8 \times 10^{-4} \\
0.13 \\
2 \cdot 1 \times 10^{-4} \\
1.1 \\
4 \cdot 1 \times 10^{-5} \\
4 \cdot 1 \times 10^{-5} \\
0.036 \\
2.0 \times 10^{-4} \\
0.71 \\
1.5 \times 10^{-5}\end{array}$ \\
\hline $\mathbf{a}^{3} \mathbf{P}-\mathbf{a}^{3} \mathbf{P}$ & $\begin{array}{l}0-1 \\
1-2\end{array}$ & & $\begin{array}{l}7.5 \times 10^{-3} \\
0.047\end{array}$ & & $\begin{array}{l}0.014 \\
0.045\end{array}$ \\
\hline$a^{5} D-a^{3} H$ & $4-4$ & $4881 \cdot 1$ & $4.8 \times 10^{-3}$ & $4227 \cdot 5$ & $1.1 \times 10^{-3}$ \\
\hline$a^{3} \mathbf{H}-\mathbf{a}^{3} \mathbf{H}$ & $\begin{array}{l}4-5 \\
5-6\end{array}$ & & $\begin{array}{l}1.9 \times 10^{-4} \\
4.1 \times 10^{-4}\end{array}$ & & $\begin{array}{l}6.5 \times 10^{-4} \\
5.8 \times 10^{-4}\end{array}$ \\
\hline$a^{5} D-a^{3} F$ & $\begin{array}{l}0-2 \\
1-2 \\
2-2 \\
3-2 \\
4-2 \\
1-3 \\
2-3 \\
3-3 \\
4-3 \\
2-4 \\
3-4 \\
4-4\end{array}$ & $\begin{array}{l}4799 \cdot 4 \\
4777 \cdot 9 \\
4733 \cdot 9 \\
4667 \cdot 0 \\
4573 \cdot 8 \\
4814 \cdot 1 \\
4769 \cdot 6 \\
4701 \cdot 6 \\
4607 \cdot 1 \\
4824 \cdot 2 \\
4754 \cdot 8 \\
4658 \cdot 1\end{array}$ & $\begin{array}{l}9.3 \times 10^{-6} \\
0.049 \\
0.10 \\
0.026 \\
2.1 \times 10^{-6} \\
1.1 \times 10^{-5} \\
0.087 \\
0.27 \\
0.038 \\
7.6 \times 10^{-6} \\
0.081 \\
0.44\end{array}$ & $\begin{array}{l}3735 \cdot 7 \\
3756 \cdot 1 \\
3795 \cdot 2 \\
3851 \cdot 4 \\
3924 \cdot 2 \\
3744 \cdot 8 \\
3783 \cdot 6 \\
3839 \cdot 5 \\
3911 \cdot 9 \\
3764 \cdot 4 \\
3819 \cdot 8 \\
3891 \cdot 3\end{array}$ & $\begin{array}{l}2.2 \times 10^{-5} \\
0.10 \\
0.20 \\
0.047 \\
1.5 \times 10^{-6} \\
8.6 \times 10^{-6} \\
0.16 \\
0.40 \\
0.066 \\
8.6 \times 10^{-7} \\
0.16 \\
0.74\end{array}$ \\
\hline$a^{5} D-a^{3} G$ & $\begin{array}{l}3-3 \\
4-4\end{array}$ & $\begin{array}{l}4046 \cdot 4 \\
4008 \cdot 4\end{array}$ & $\begin{array}{l}8.0 \times 10^{-3} \\
0.019\end{array}$ & $\begin{array}{l}3445 \cdot 4 \\
3463 \cdot 4\end{array}$ & $\begin{array}{l}0.017 \\
0.032\end{array}$ \\
\hline$a^{5} D-a^{3} D$ & $\begin{array}{l}0-1 \\
1-1 \\
1-2 \\
2-2 \\
3-2 \\
2-3 \\
3-3 \\
4-3\end{array}$ & $\begin{array}{l}3366 \cdot 2 \\
3355 \cdot 6 \\
3356 \cdot 6 \\
3334 \cdot 9 \\
3301 \cdot 6 \\
3319 \cdot 3 \\
3286 \cdot 2 \\
3239 \cdot 7\end{array}$ & $\begin{array}{l}0.13 \\
0.15 \\
0.095 \\
0.11 \\
0.027 \\
0.044 \\
0.047 \\
0.23\end{array}$ & $\begin{array}{l}? \\
? \\
? \\
? \\
? \\
? \\
? \\
?\end{array}$ & $\begin{array}{l}0.22 \\
0.19 \\
0.20 \\
0.18 \\
0.11 \\
0.097 \\
0.089 \\
0.37\end{array}$ \\
\hline
\end{tabular}

* From Garstang (1957), where results are given for many additional lines. 
Table 10

Transition probabilities for selected* lines of Fe II

\begin{tabular}{|c|c|c|c|}
\hline Transition & $\mathbf{J}-\mathbf{J}^{\prime}$ & $\lambda$ & $A$ \\
\hline$a^{6} D-a^{6} S$ & $\begin{array}{r}4 \frac{1}{2}-2 \frac{1}{2} \\
3 \frac{1}{2}-2 \frac{1}{2} \\
2 \frac{1}{2}-2 \frac{1}{2} \\
1 \frac{1}{2}-2 \frac{1}{2} \\
\frac{1}{2}-2 \frac{1}{2}\end{array}$ & $\begin{array}{l}4287 \cdot 4 \\
4359 \cdot 3 \\
4413 \cdot 8 \\
4452 \cdot 1 \\
4474 \cdot 9\end{array}$ & $\begin{array}{l}1.12 \\
0.82 \\
0.58 \\
0.37 \\
0.18\end{array}$ \\
\hline$a^{4} F-a^{4} G$ & $\begin{array}{l}4 \frac{1}{2}-5 \frac{1}{2} \\
3 \frac{1}{2}-5 \frac{1}{2} \\
4 \frac{1}{2}-4 \frac{1}{2} \\
3 \frac{1}{2}-4 \frac{1}{2} \\
2 \frac{1}{2}-4 \frac{1}{2} \\
4 \frac{1}{2}-3 \frac{1}{2} \\
3 \frac{1}{2}-3 \frac{1}{2} \\
2 \frac{1}{2}-3 \frac{1}{2} \\
1 \frac{1}{2}-3 \frac{1}{2} \\
4 \frac{1}{2}-2 \frac{1}{2} \\
3 \frac{1}{2}-2 \frac{1}{2} \\
2 \frac{1}{2}-2 \frac{1}{2} \\
1 \frac{1}{2}-2 \frac{1}{2}\end{array}$ & $\begin{array}{l}4244 \cdot 0 \\
4346 \cdot 9 \\
4177 \cdot 2 \\
4276 \cdot 8 \\
4352 \cdot 8 \\
4146 \cdot 6 \\
4244 \cdot 8 \\
4319 \cdot 6 \\
4372 \cdot 4 \\
4134 \cdot 0 \\
4231 \cdot 6 \\
4305 \cdot 9 \\
4358 \cdot 4\end{array}$ & $\begin{array}{l}0.90 \\
0.21 \\
0.14 \\
0.65 \\
0.31 \\
8.7 \times 10^{-3} \\
0.25 \\
0.53 \\
0.28 \\
2.0 \times 10^{-4} \\
0.024 \\
0.31 \\
0.73\end{array}$ \\
\hline$a^{4} F-b^{4} F$ & $\begin{array}{l}4 \frac{1}{2}-4 \frac{1}{2} \\
3 \frac{1}{2}-4 \frac{1}{2} \\
2 \frac{1}{2}-4 \frac{1}{2} \\
4 \frac{1}{2}-3 \frac{1}{2} \\
3 \frac{1}{2}-3 \frac{1}{2} \\
2 \frac{1}{2}-3 \frac{1}{2} \\
1 \frac{1}{2}-3 \frac{1}{2} \\
4 \frac{1}{2}-2 \frac{1}{2} \\
3 \frac{1}{2}-2 \frac{1}{2} \\
2 \frac{1}{2}-2 \frac{1}{2} \\
1 \frac{1}{2}-2 \frac{1}{2} \\
3 \frac{1}{2}-1 \frac{1}{2} \\
2 \frac{1}{2}-1 \frac{1}{2} \\
1 \frac{1}{2}-1 \frac{1}{2}\end{array}$ & $\begin{array}{l}4814 \cdot 6 \\
4947 \cdot 4 \\
5049 \cdot 3 \\
4774 \cdot 7 \\
4905 \cdot 3 \\
5005 \cdot 5 \\
5076 \cdot 6 \\
4745 \cdot 5 \\
4874 \cdot 5 \\
4973 \cdot 4 \\
5043 \cdot 5 \\
4852 \cdot 7 \\
4950 \cdot 7 \\
5020 \cdot 2\end{array}$ & $\begin{array}{l}0.40 \\
0.050 \\
7.2 \times 10^{-4} \\
0.13 \\
0.22 \\
0.071 \\
1.6 \times 10^{-5} \\
0.013 \\
0.17 \\
0.14 \\
0.065 \\
0.022 \\
0.17 \\
0.18\end{array}$ \\
\hline$a^{6} D-b^{4} F$ & $\begin{array}{r}4 \frac{1}{2}-4 \frac{1}{2} \\
3 \frac{1}{2}-4 \frac{1}{2} \\
2 \frac{1}{2}-4 \frac{1}{2} \\
4 \frac{1}{2}-3 \frac{1}{2} \\
3 \frac{1}{2}-3 \frac{1}{2} \\
2 \frac{1}{2}-3 \frac{1}{2} \\
1 \frac{1}{2}-3 \frac{1}{2} \\
4 \frac{1}{2}-2 \frac{1}{2} \\
3 \frac{1}{2}-2 \frac{1}{2} \\
2 \frac{1}{2}-2 \frac{1}{2} \\
1 \frac{1}{2}-2 \frac{1}{2} \\
\frac{1}{2}-2 \frac{1}{2} \\
3 \frac{1}{2}-1 \frac{1}{2} \\
2 \frac{1}{2}-1 \frac{1}{2} \\
1 \frac{1}{2}-1 \frac{1}{2} \\
\frac{1}{2}-1 \frac{1}{2}\end{array}$ & $\begin{array}{l}4416 \cdot 3 \\
4492 \cdot 6 \\
4550 \cdot 5 \\
4382 \cdot 8 \\
4458 \cdot 0 \\
4514 \cdot 9 \\
4555 \cdot 0 \\
4358 \cdot 1 \\
4432 \cdot 5 \\
4488 \cdot 8 \\
4528 \cdot 4 \\
4552 \cdot 0 \\
4414 \cdot 5 \\
4470 \cdot 3 \\
4509 \cdot 6 \\
4533 \cdot 0\end{array}$ & $\begin{array}{l}0.46 \\
0.060 \\
2.6 \times 10^{-7} \\
0.055 \\
0.29 \\
0.066 \\
4.8 \times 10^{-8} \\
1.6 \times 10^{-5} \\
0.054 \\
0.15 \\
0.046 \\
2.0 \times 10^{-6} \\
5.9 \times 10^{-6} \\
0.029 \\
0.058 \\
0.016\end{array}$ \\
\hline
\end{tabular}

* From Garstang (1962), where data for many other lines may be found. 
neon references are given to the original papers containing the data we have quoted. In each case we have quoted what we believe to be the best available results.

In a few cases we have given original data calculated for inclusion here. $\mathrm{Mg}$ VI and K v were originally calculated by Pasternack (1940). We have recalculated these, using the best available technique. The principal change results from the use of improved quadrupole radial integrals, which we have estimated by extrapolation. The changes

\section{Table 11}

Transition probabilities for some miscellaneous lines

\begin{tabular}{|c|c|c|c|c|c|}
\hline Ion & Transition & $\mathbf{J}-\mathbf{J}^{\prime}$ & $\lambda$ & $A$ & Notes \\
\hline $\mathrm{Mg}_{\mathrm{I}}$ & $3 s^{2} 1 S-3 s 3 p{ }^{3} P$ & $0-2$ & $4562 \cdot 5$ & $2.0 \times 10^{-4}$ & 1 \\
\hline NiIII & $\begin{array}{l}{ }^{3} \mathrm{~F}-{ }^{3} \mathrm{P} \\
{ }^{3} \mathrm{~F}-{ }^{3} \mathrm{P} \\
{ }^{3} \mathrm{~F}-{ }^{3} \mathrm{P} \\
{ }^{3} \mathrm{~F}-{ }^{1} \mathrm{G}\end{array}$ & $\begin{array}{l}3-1 \\
2-0 \\
3-2 \\
3-4\end{array}$ & $\begin{array}{l}6401 \cdot 5 \\
6682 \cdot 2 \\
6533 \cdot 7 \\
4596 \cdot 8\end{array}$ & $\begin{array}{l}0.038 \\
0.046 \\
0.12 \\
0.18\end{array}$ & 2 \\
\hline $\mathrm{C}_{\mathrm{II}}$ & $2 p{ }^{2} P$ & $\frac{1}{2}-1 \frac{1}{2}$ & $156 \mu$ & $2.4 \times 10^{-6}$ & 3 \\
\hline NiII & $2 p{ }^{2} \mathbf{P}$ & $\frac{1}{2}-1 \frac{1}{2}$ & $57 \cdot 3 \mu$ & $4.8 \times 10^{-5}$ & 3 \\
\hline OIV & $2 p{ }^{2} P$ & $\frac{1}{2}-1 \frac{1}{2}$ & $25.9 \mu$ & $5 \cdot 2 \times 10^{-4}$ & 3 \\
\hline $\mathrm{Ne} I I$ & $2 p^{52} P$ & $1 \frac{1}{2}-\frac{1}{2}$ & $12.8 \mu$ & 0.0086 & 3 \\
\hline Mgiv & $2 p^{52} P$ & $1 \frac{1}{2}-\frac{1}{2}$ & $4.49 \mu$ & 0.20 & 4 \\
\hline Si II & $3 p^{2} P$ & $\frac{1}{2}-1 \frac{1}{2}$ & $34.8 \mu$ & $2 \cdot 1 \times 10^{-4}$ & 4 \\
\hline Siv & $3 p^{2} P$ & $\frac{1}{2}-1 \frac{1}{2}$ & $10.6 \mu$ & 0.0077 & 4 \\
\hline
\end{tabular}

1 This line arises partly from magnetic quadrupole radiation and partly from nuclear-spin-induced electric dipole radiation. See Garstang (1967).

2 Possible identification of 6401.5 by Flather and Osterbrock (1960). Other Ni III lines have not been seen in gaseous nebulae. Transition probabilities from Garstang (1958).

${ }^{3}$ From Wiese et al. (1966).

4 Calculated by Garstang for inclusion here.

in the magnetic dipole results and in the relative electric quadrupole results are fairly small. We have also revised Pasternack's (1940) results for MnVI, FevII, Mnv and Fevi by introducing revised quadrupole radial integrals as described by Garstang (1964). We included Mgvi because of its possible, as yet unconfirmed, identification by Gauzit (1966). In Table 11 we have included some infrared transitions; other such transitions appear in many of the other tables, or can be found in the references (e.g. for FeII and other heavy ions).

\section{Acknowledgment}

The preparation of this paper was supported by the National Science Foundation under Grant GP-6595. 


\section{References}

Bowen, I.S. (1955) Astrophys. J., 121, 306.

Bowen, I.S. (1960) Astrophys. J., 132, 1.

Czyzak, S.J., Krueger, T.K. (1963) Mon. Not. R. astr. Soc., 126, 177, with an important correction in 1965, ibid., 129, 103.

Flather, E., Osterbrock, D.E. (1960) Astrophys. J., 132, 18.

Garstang, R.H. (1957) Mon. Not. R. astr. Soc., 117, 393.

Garstang, R.H. (1958) Mon. Not. R. astr. Soc., 118, 234.

Garstang, R.H. (1962) Mon. Not. R. astr. Soc., 124, 321.

Garstang, R.H. (1964) J. Res. nat. Bur. Stand. Sec. A., 68, 61.

Garstang, R.H. (1967) Astrophys. J., 148, 579.

Gauzit, J. (1966) C. r. hebd. Séanc. Acad. Sci. Paris, 262, 523.

Malville, J.M., Berger, R.A. (1965) Planet. Space Sci., 13, 1131.

Pasternack, S. (1940) Astrophys. J., 92, 129.

Wiese, W.L., Smith, M.W., Glennon, B.M. (1966) Atomic Transition Probabilities, Vol. I: Hydrogen through Neon, Nat. Stand. Ref. Data Series - Nat. Bur. Stand. 4, U.S. Government Printing Office, Washington, D.C.

\section{DISCUSSION}

Garstang: I would like to open the discussion myself by asking the question I am frequently asked - Do I believe the results? The answer is - Yes, I do. While one cannot entirely exclude the possibility that some unsuspected configuration interaction may produce significant perturbations, this seems unlikely for the transitions of interest for forbidden lines. There is now some substantial evidence for the basic correctness of the results. The comparison of [Fe II] lines in $\eta$ Carinae with theoretical values (Thackeray, Mon. Not. R. astr. Soc., 135, 1967, 23) shows astonishingly good agreement, confirming the broad overall accuracy of the relative line strengths. Recent experimental work on interference effects in Zeeman components of two lines of mixed magnetic-dipole and electricquadrupole radiation of $[\mathrm{PbI}]$ and $[\mathrm{Pb} \mathrm{II}]$ by Hults (J. opt. Soc. Am., 56, 1966, 1298) shows excellent agreement with the relative contributions of the two kinds of radiation predicted by Garstang (J. Res. nat. Bur. Stand., Sec. A, 68, 1964, 61). Finally, experiments (Husain and Wiesenfeld, Nature, $213,1967,1227)$ on flash photolysis of trifluoro-iodomethane have led to an estimate of the lifetime of the upper state of the lowest doublet in II within a factor 3 of the theoretical lifetime given by Garstang (J. Res. nat. Bur. Stand., Sec. A, 68, 1964, 61). In view of the experimental difficulties in handling a state whose lifetime is of the order of $0.1 \mathrm{sec}$ this agreement must be considered satisfactory. Taking all these results together I think we must regard the transition probabilities of forbidden lines as reasonably well established.

Menzel: Some mention has been made of high-level transitions in hydrogen. I have derived an asymptotic formula for the $f$-values of such transitions between levels of quantum numbers $n$ and $n^{\prime}$ with $n-n^{\prime}=c$.

The $f$-value is

$$
f_{n n^{\prime}}=\frac{4 n^{\prime}}{3 c^{2}} J_{\mathrm{c}}(c) J_{\mathrm{c}}(c)=n^{\prime} M(c),
$$

where $J_{\mathrm{c}}(c)$ and $J_{\mathrm{c}}{ }^{\prime}(c)$ are respectively the Bessel functions of equal argument and order and its derivative. Examples of $M(c)$ follow:

$$
\begin{array}{ll}
M(1)=1.9077 \times 10^{-1}, & M(3)=8.1056 \times 10^{-3} \\
M(2)=2.6332 \times 10^{-2}, & M(4)=3.4917 \times 10^{-3} .
\end{array}
$$

Note that for $n^{\prime}=100, f_{101,100}-19$. The question might be asked, How can one reconcile such large $f$-values with the well-known $f$-sum rule $f=1$ ? The chief point is that one must sum over 
all transitions, both upward and downward, from level $n^{\prime}$. The former are counted positive, the latter, negative. Downward $f$-values can be calculated from upward ones by the formula $f_{n}{ }^{\prime \prime} n^{\prime}=$ $-n^{\prime \prime} f_{n^{\prime}} n^{\prime \prime} / n^{\prime 2}$. With these formulas, the negative contributions nearly cancel the large positive contributions, leaving the exact remainder

$$
4 \sum_{c=1}^{\infty} \frac{J_{c}(c) J_{c}{ }^{\prime}(c)}{c}=1
$$

a new theorem in the theory of Bessel functions. 